\title{
The efficacy and safety of levetiracetam in the prophylaxis of migraine headaches in children - a randomised trial
}

\author{
Mohammadreza Ghazavi ${ }^{1}$, Gholamreza Jelodar ${ }^{1,2}$, Omid Yaghini' ${ }^{1}$ Jafar Nasiri', \\ Saeid Sadeghian'2, Reza Azizi Malamiri² \\ 'Department of Paediatric Neurology and Child Growth and Development Centre, School of Medicine, Isfahan University \\ of Medical Sciences, Isfahan, Iran \\ 2Department of Paediatric Neurology, Golestan Medical, Educational, and Research Centre, Ahvaz Jundishapur University \\ of Medical Sciences, Ahvaz, Iran
}

\section{ABSTRACT}

Introduction: Migraine headaches have a negative impact on daily living activities in children. Many children with migraine need prophylactic medications in addition to biobehavioural measures. Until now, only topiramate has been approved by the FDA (US Food and Drug Administration) as a prophylactic medication in children with migraine. However, topiramate has many unacceptable adverse cognitive effects, therefore, conducting well-designed trials to show the efficacy of safer medications has been recommended.

Aim of the study: We conducted a trial to assess the efficacy and safety of levetiracetam in the prophylaxis of migraine headaches in children.

Material and methods: In an open-label, randomised, controlled trial, we assessed the efficacy and safety of levetiracetam in the prophylaxis of migraine headaches in children compared to a well-known prophylactic agent, amitriptyline. The primary outcome measure was reduction of monthly headache frequency. The secondary outcome measures were reduction in headache duration and severity. In addition, we studied the effect of these medications on headache disability score using the Paediatric Migraine Disability Assessment (PedMIDAS) questionnaire, as well as their adverse side effects. The trial was continued for three months after administering the medications.

Results: We enrolled 66 children with migraine, and 60 of them completed the study (30 in each arm). Ninety per cent $(27 / 30)$ of children in each arm showed more than $50 \%$ reduction in monthly headache frequency. Duration and severity of headaches were also significantly decreased at the end of the trial. In addition, the safety profile of medications was acceptable, and headache disability score was significantly improved at the end of the trial.

Conclusions: These results indicate that levetiracetam might be effective and safe in the prophylaxis of migraine headaches in children. It deserves to be considered in the prophylaxis of migraine headaches in children.

\section{KEY WORDS:}

children, prophylaxis, migraine, levetiracetam, amitriptyline.

\section{ADDRESS FOR CORRESPONDENCE:}

Reza Azizi Malamiri, Department of Paediatric Neurology, Golestan Medical, Educational, and Research Centre, Ahvaz Jundishapur University of Medical Sciences, \#90, Golestan Blvd., P.O. Box 6135733118, Ahvaz, Iran, ORCID: 0000-0001-5423-6375, e-mail: azizi.ramin@gmail.com 


\section{INTRODUCTION}

Migraine is common among children and adolescents. According to epidemiological studies, about $10 \%$ of children and $28 \%$ of adolescents experience these headaches. Many children with migraine headaches experience social isolation and lose many school days. In addition, these headaches have a severe impact on different aspects of quality of life of children and their families. Therefore, effective management of these headaches is required [1-3].

By improving the quality and quantity of sleep, having appropriate and timely meals, living in a safe and calm environment, and using proper analgesics or NSAIDs, a large number of children with migraine could manage their headaches without using daily prophylactic agents. However, these measures are not effective in a significant portion of children with migraine and they need daily prophylactic agents. According to the consensus, children with migraine headaches need daily prophylactic agents if they have the following criteria: at least four migraine attacks per month, especially when headaches have moderate to severe intensity or they last more than four hours, or they have poor response to acute treatments [1].

Many studies have shown the efficacy and safety of different medications in the prophylaxis of migraine headaches in children, even though until now only topiramate has FDA approval as a prophylactic agent in children with migraine [4]. Interestingly, results of the Childhood and Adolescent Migraine Prevention (CHAMP) trial indicate that both topiramate and amitriptyline are not more effective than placebo in reduction of headache frequency or headache-related disability in childhood and adolescent migraine [5]. In addition, many studies have been concluded that the placebo effect in both adult and child migraine is high, and the placebo effect should be considered in every new study [5].

To select a medication as a prophylactic agent in children with migraine the medication should not only reduce both the frequency and severity of headaches effectively, but must also have the fewest adverse effects [1]. Topiramate might be effective in reducing the frequency and severity of headaches but unfortunately has significant negative cognitive effects such as inattention, memory problems, information processing speed disorders, and verbal fluency difficulties in both children and adults [6]. Therefore, new effective agents without serious adverse effects have to be studied in children with migraine. Recently, a number of studies have indicated the efficacy and safety of levetiracetam as a prophylactic agent in both adults and children with migraine [2,7-12].

Levetiracetam is an anticonvulsive agent that has different mechanisms of action. Levetiracetam has been approved by the FDA to be used in both focal and generalised seizures in adults and children [12]. It has only a few adverse side effects such as somnolence and mood disorders, particularly irritability, which can easily be managed by decreasing the dose. Although some studies have shown its efficacy and safety in the prophylaxis of migraine headaches in adults and children, a recent systematic review concluded that the role of levetiracetam for the prophylactic treatment of chronic migraine is not well established and further well-designed studies need to be conducted [12].

In our country, Iran, levetiracetam is available in forms of tablet and syrup, and almost all health insurance companies support its usage. In addition, to our knowledge, no controlled study with an acceptable sample size has been conducted in our country to show its efficacy and safety as a prophylactic agent in children with migraine. Therefore, we carried out a randomised, controlled trial to assess its efficacy and safety in children with migraine as a prophylactic agent.

\section{MATERIAL AND METHODS}

\section{STUDY DESIGN AND LOCATION}

We conducted a randomised, parallel-group controlled, open-label trial to assess the efficacy and safety of levetiracetam in children with migraine as a prophylactic agent. After reviewing of the trial design, the review board of the centre did not agree with a placebo arm as the control group, so we administered another well-recognised migraine prophylactic agent, amitriptyline. As we mentioned earlier, the CHAMP trial showed that the effect of amitriptyline in children with migraine is not more than placebo [5]. Therefore, we administered amitriptyline in the control group to get the board's opinion. The study was conducted at Imam Hossein Children's Hospital, Isfahan, a tertiary university-affiliated children's hospital located almost in the centre of Iran, and provides medical services not only to Isfahan province but also to eight provinces with a population of more than six million. This study was approved and registered with the Iranian Registry of Clinical Trials, number IRCT20171212037850N1. The study was conducted between October 2017 and July 2018.

\section{PARTICIPANTS, AND INCLUSION AND EXCLUSION CRITERIA}

All children aged between five and 15 years with migraine headaches according to the International Classification of Headache Disorders, third edition, were eligible for recruitment [13]. We included all those children with migraine headaches with and without aura, who had at least four attacks of headache per month that either lasted at least two hours or had moderate to severe intensity. Recruited children had had headaches for at least six months before enrolment and had not been administered a migraine prophylactic agent. We excluded all those children who had the following criteria: epilepsy, complicated migraine, history of adverse reactions to 
tricyclic antidepressants, children with severe psychiatric problems like depression and ADHD, and those who were taking analgesics more than four times per week to control migraine attacks. We also excluded all those who were unable to record the frequency, duration, and severity of headaches, or had been administered migraine prophylactic agents.

\section{RANDOMISATION}

All children who were eligible for recruitment and their parents were interviewed, and after obtaining written, informed consent they were randomly assigned to levetiracetam or amitriptyline groups. The randomisation was performed using a random number table, and all the consecutive patients were randomised to allocate into quaternary blocks. After randomisation, the medications in generic form were taken to the participants using packages made by central pharmacy of the centre. These packages had identical shape and colour, and their contents were provided by the central pharmacy of the centre. After opening the packages, the parents could see the instructions for taking the medications.

\section{INTERVENTION AND DOSAGES OF THE MEDICATIONS}

Each child was randomised into either the levetiracetam or the amitriptyline group. In this study, we used generic forms of levetiracetam and amitriptyline. In both groups, the dose of the medication administered was based on the body weight. In the levetiracetam group, the starting dose of levetiracetam was $10 \mathrm{mg} / \mathrm{kg} / \mathrm{day}$ (divided in two doses q $12 \mathrm{~h}$ ) and increased to $20 \mathrm{mg} / \mathrm{kg} /$ day (divided in two doses q $12 \mathrm{~h}$ ) after one week. In the amitriptyline group, the starting dose was $0.25 \mathrm{mg} / \mathrm{kg} /$ day (bedtime), and this was increased by $0.25 \mathrm{mg} / \mathrm{kg} /$ day weekly to final dose of $1 \mathrm{mg} / \mathrm{kg} /$ day (bedtime). The instructions for using medications were carefully written in plain language and placed inside the packages.

\section{OUTCOME MEASURES}

The primary outcome measure was the effect of the medications on the frequency of headaches per month, and more than $50 \%$ reduction in headache frequency compared to baseline was considered as the significance cut-off point. The secondary outcome measure was the effect of the medications on the severity and duration of headaches. More than 50\% reduction in the duration and severity of headaches was considered significant. To assess these outcomes correctly, we divided the trial into two phases. In the first phase of the study, which lasted one month for each participant, all the children and their parents were carefully instructed by trained interviewing staff to accurately record the frequency (on a monthly basis), duration (in hours), and severity (using a scale) of headaches in the prepared charts that were given to them. To ensure a better objective measure of the severity of headaches we used the Wong-Baker Faces Pain Rating Scale. Skilled staff instructed the children and their parents to familiarise them with this scale and how to use it. The validity and reliability of this scale have been confirmed in our population in previous studies [14]. At the end of the first phase of the study and after obtaining written, informed consent, all the children who had the inclusion criteria were randomised to receive one of the medications. After randomisation, all the participants were followed for three months more and an interviewing staff member called them at weekly intervals to assess their adherence to the study protocol. In the second phase of the trial, all the children and their parents were asked to accurately record the frequency, duration, and severity of headaches in the prepared charts that were given to them. We also measured the disability score of the participants using the PedMIDAS questionnaire. The validity and reliability of this scale have been confirmed in our population in previous studies [15]. All the children and their parents were visited and interviewed at the end of each month after randomisation. All the children and their parents were also asked to accurately record any adverse effects after taking the medications, especially any sedation, irritability, and changes in appetite.

\section{STATISTICS}

According to previous studies, and assuming a 50\% reduction in headache frequency in the amitriptyline group and $65 \%$ reduction in headache frequency in levetiracetam group and the required power of $80 \%$, the sample size was calculated to be 32 in each study arm [14]. All the data including the frequency, duration, severity of headaches, and PedMIDAS scores were carefully recorded in prepared charts and then entered into a central computerised database. All the quantitative data were tested for normality using the Shapiro-Wilk test. Normal data were described using mean $\pm S \mathrm{D}$, and non-normal data were described using median and range. The differences between two study arms were tested using independent sample $t$-test when data were distributed normally, otherwise we used non-parametric equivalent tests. Quantitative data were analysed using $\chi^{2}$ test. In all statistical assessments, $p<0.05$ was considered significant. To reduce the bias in data analysis, a biostatistician who was not aware of the study groups analysed all data.

\section{ETHICS}

The review board of the centre approved the study protocol, and we performed the study in accordance with the ethical standards as laid down in the 1964 Declaration of Helsinki and its later amendments. All the parents 
read and signed written, informed consent forms before enrolment.

\section{RESULTS}

\section{BASELINE CHARACTERISTICS}

During the first period of the trial about 100 children with migraine were eligible for recruitment, but we could obtain written, informed consent from only 66 of them, who were randomised to one of the trial groups (34 children were in the levetiracetam arm and 32 in the amitriptyline arm). From these 66 children, 60 completed the trial. We lost two children because of relocation to another part of the country and four children because of non-adherence to the trial protocol. Of note, none of children was lost to follow-up because of the adverse effects of the medications. Table 1 shows the baseline characteristics of the children in both groups. As has been shown, both groups had no significant difference in terms of gender, age, headache frequency and severity, and PedMIDAS score. However, the median headache duration in the first period of the study was significantly longer in the levetiracetam group ( $p=0.031$, Mann-Whitney rank sum test) (Table 1).

\section{EFFICACY OF THE MEDICATIONS}

The main efficacy measure of the medications was the effect of them on the frequency of headaches during the trial period. Analysis of the results indicated that compared to the baseline and at the end of the trial, in both groups, the medications reduced the headache frequency per month by more than $50 \%$ in $90 \%$ (27/30) of children. The other efficacy measures of the medications were the effect of them on the duration and severity of headaches. In the levetiracetam group, the duration and severity of headaches were reduced by more than $50 \%$ in $90 \%$ $(27 / 30)$ of children. In the amitriptyline group, the dura-

TABLE 1. Baseline characteristics of the both groups in the first period of the trial

\begin{tabular}{|l|c|c|}
\hline Characteristics & $\begin{array}{c}\text { Levetiracetam } \\
(n=30)\end{array}$ & $\begin{array}{c}\text { Amitriptyline } \\
(n=30)\end{array}$ \\
\hline Gender & $\begin{array}{c}\text { Boys: } \\
16(53.3 \%)\end{array}$ & $\begin{array}{c}\text { Boys: } \\
21(30 \%)\end{array}$ \\
\hline Age: mean \pm SD & $10.6 \pm 2.6$ & $9.6 \pm 2.6$ \\
\hline $\begin{array}{l}\text { Headache frequency } \\
\text { (per month), median (range) }\end{array}$ & $12(4-30)$ & $11(4-30)$ \\
\hline $\begin{array}{l}\text { Headache duration } \\
\text { (in hours), median (range) }\end{array}$ & $4.5(1-72)$ & $2(1-24)$ \\
\hline $\begin{array}{l}\text { Headache severity, median } \\
\text { (range) }\end{array}$ & $8(4-10)$ & $8(4-10)$ \\
\hline PedMIDAS, median (range) & $66(12-102)$ & $52(12-109)$ \\
\hline
\end{tabular}

tion of headaches was reduced by more than $50 \%$ in $90 \%$ $(27 / 30)$ of children. However, the headache severity was reduced by more than $50 \%$ in $80 \%$ (24/30) of children. These results indicated that both medications are effective in controlling of the frequency, duration, and severity of headaches in children with migraine as prophylactic agents after a three-month period at the mentioned dosages. We also measured the PedMIDAS disability score, and the results indicated that the median PedMIDAS score was significantly improved in both groups during the trial, and at the end of the third month after taking medications, the median PedMIDAS score in both groups was 0 , compared to the first period of the trial (Table 1) ( $p<0.001$, Mann-Whitney rank sum test).

\section{ADVERSE EFFECTS}

During the study, children and their parents reported a number of side effects; however, none of these adverse effects led to withdrawal of the medication by the children. In the levetiracetam group, five children developed irritability and bad temperament that had an impact on the family condition; however, no dosage adjustment was required. All the parents reported that irritability and bad temperament disappeared during the trial. In the amitriptyline group, six children developed drowsiness in the first month after taking the medication, which disappeared during follow-up.

\section{DISCUSSION}

Results of this open-label, randomized, parallel, group-controlled trial indicate that both levetiracetam and amitriptyline might be effective as prophylactic agents in a highly selected group of children with uncomplicated migraine. Evidence of efficacy is that both of these medications after a three-month period and at the mentioned dosages significantly reduced the frequency of headaches as the primary outcome measure in $90 \%$ of our children (27/30). They also significantly reduced the duration and severity of headaches in both groups at the end of the trial. Our results also indicate that both of these medications could improve migraine disability score after a three-month period. Evidence of improved disability score is that both of these medications significantly reduced the median PedMIDAS scores from 66 (12-102) in the levetiracetam group and 52 (12-109) in the amitriptyline group to almost zero at the end of the trial. In addition, our results indicate that both of these medications are safe. Evidence of safety is that none of the children discontinued the medication because of adverse effects; however, a number of our participants reported bad temperament in the levetiracetam group and drowsiness in the amitriptyline group.

Levetiracetam has been approved by FDA as an antiseizure medication in both focal and generalised seizures. 
In addition, a number of studies in both adults and children showed the efficacy of levetiracetam as an effective and safe prophylactic agent [7-12]. It has different mechanisms of action, but the main mechanism of action in migraine prophylaxis has not been defined yet. Moreover, a systematic review has concluded that levetiracetam could be considered as a prophylactic agent in patients with migraine, but the authors of this systematic review have recommended conducting well-designed studies to show its efficacy and safety in children [12].

To our knowledge, only three studies have been conducted in children to show the efficacy of levetiracetam as a migraine prophylactic agent. Two of the studies were retrospective and were based on chart review $[7,16]$. Both of these studies had small sample sizes. The third study was conducted by Pakalnis et al. in children [9]. In this open-label, uncontrolled study 22 children were enrolled and 20 completed the study. They were aged between seven and 16 years. The starting dose was $20 \mathrm{mg} / \mathrm{kg} /$ day and was increased to $40 \mathrm{mg} / \mathrm{kg} /$ day in the second month of the follow-up. The study was continued for two months after administering the medication. At the end of the study, 90\% (18/20) of children showed more than $50 \%$ reduction in monthly headache frequency. In addition, PedMIDAS score were significantly improved. The results of this study are in accordance with ours. In addition, our study was a randomised, controlled study with a reasonable sample size.

In this study, we decided to administer levetiracetam by $20 \mathrm{mg} / \mathrm{kg} /$ day (divided in two doses $\mathrm{q} 12 \mathrm{~h}$ ) as a maintenance dosage. We could administer levetiracetam at higher dosages as in previous studies, but we know that levetiracetam at higher dosages could have more side effects, especially irritability and behavioural problems. In addition, our results indicate that levetiracetam $20 \mathrm{mg} /$ $\mathrm{kg} /$ day (divided in two doses q $12 \mathrm{~h}$ ) is effective in $90 \%$ of children with uncomplicated migraine and is safe. Therefore, it seems that $20 \mathrm{mg} / \mathrm{kg} /$ day (divided in two doses $\mathrm{q} 12 \mathrm{~h}$ ) is an acceptable dosage of levetiracetam as a prophylactic medication in children with migraine.

As mentioned in the introduction section, in order to select a medication as a prophylactic agent in children with migraine, that medication not only should reduce both the frequency and severity of headaches effectively but must also have minimal adverse effects. According to the previous studies and our study, levetiracetam could be considered as an effective and safe prophylactic medication in children with migraine.

Although until know only topiramate has been approved by the FDA as a prophylactic medication in children with migraine, previous studies showed the efficacy of a number of anticonvulsive medications in children with migraine. The main medication among these effective agents is sodium valproate. Sodium valproate is a broad-range anticonvulsant and has different mechanisms of action. The main mechanism of action in $\mathrm{mi}-$ graine prophylaxis is unknown, but different mechanisms have been proposed. Sodium valproate could increase gamma-aminobutyric acid (GABA) levels, and higher GABA levels could suppress migraine related events in the cortex. Other proposed mechanisms are inhibition of voltage-sensitive calcium channels (T-type), reducing the inflammatory effect of serotoninergic neurons, thus reducing the central trigeminal nerve activation $[17,18]$. Studies in children and adults have indicated its efficacy in migraine prophylaxis, but adverse effects such as tiredness, drowsiness, dizziness, tremor, hair loss, skin rash, and nausea, and especially weight gain, limit its usage.

The results of our study should be interpreted in the face of certain limitations. This is an open-label trial. In addition, because of ethical issues our board did not agree with a placebo arm; however, we tried to control our results by administering a well-known migraine prophylactic agent, amitriptyline. As mentioned earlier, the CHAMP trial showed that amitriptyline is not more effective than placebo in reduction of headache frequency or headache-related disability in childhood and adolescent migraine [5]. In our study, the children who took amitriptyline showed a $90 \%$ response. We have no explanation for these results, but we think that our sample size was very small in comparison to the CHAMP trial, and we also enrolled a group of highly selected children with migraine, and this kind of selection could lead to better response compared to placebo effect.

\section{CONCLUSIONS}

Our results indicate that levetiracetam could be a prophylactic option in reasonably selected children with uncomplicated migraine. When levetiracetam administered at a starting dosage of $10 \mathrm{mg} / \mathrm{kg} / \mathrm{q} 12 \mathrm{~h}$ and increased to $20 \mathrm{mg} / \mathrm{kg} / \mathrm{q} 12 \mathrm{~h}$, it could be effective in a three-month period in reducing the frequency, duration, and severity of headaches. Levetiracetam could also improve headache disability scores after a three-month period. In addition, its safety profile is acceptable, and adverse side effects are manageable. Therefore, it deserves to be considered in the prophylaxis of migraine headaches in children.

\section{ACKNOWLEDGMENTS}

This paper is from the thesis of Gholamreza Jelodar, MD. The Research Deputy of Isfahan University of Medical Sciences provided financial and logistic support for this study (grant no. IR.mui.rec.1396.3.781) but had no role in the study design, data collection, analysis, or interpretation, writing the report, or in the decision to submit the article for publication. The authors are grateful to all parents and patients for their participation in this study. We are extremely indebted to the authorities of the Research Deputy of Isfahan University of Medical Sciences for their financial and logistic support. 


\section{DISCLOSURE}

The authors declare no conflict of interest.

\section{REFERENCES}

1. Toldo I, De Carlo D, Bolzonella B, et al. The pharmacological treatment of migraine in children and adolescents: an overview. Expert Rev Neurother 2012; 12: 1133-1142.

2. Tajti J, Szok D, Csati A, Vecsei L. Prophylactic Drug Treatment of Migraine in Children and Adolescents: An Update. Curr Pain Headache Rep 2016; 20: 1.

3. Merison K, Jacobs H. Diagnosis and Treatment of Childhood Migraine. Curr Treat Options Neurol 2016; 18: 48.

4. Sakulchit T, Meckler GD, Goldman RD. Topiramate for pediatric migraine prevention. Can Fam Physician 2017; 63: 529-531.

5. Powers SW, Coffey CS, Chamberlin LA, et al. Trial of Amitriptyline, Topiramate, and Placebo for Pediatric Migraine. N Engl J Med 2017; 376: 115-124.

6. Sommer BR, Mitchell EL, Wroolie TE. Topiramate: Effects on cognition in patients with epilepsy, migraine headache and obesity. Ther Adv Neurol Disord 2013; 6: 211-227.

7. Awaad Y, Rizk T. Levetiracetam in the treatment of pediatric headache. J Taibah Univ Med Sci 2014; 9: 74-77.

8. Brighina F, Palermo A, Aloisio A, et al. Levetiracetam in the prophylaxis of migraine with aura: a 6-month open-label study. Clin Neuropharmacol 2006; 29: 338-342.

9. Pakalnis A, Kring D, Meier L. Levetiracetam prophylaxis in pediatric migraine - an open-label study. Headache 2007; 47: 427-430.

10. Sadeghian H, Motiei-Langroudi R. Comparison of Levetiracetam and sodium Valproate in migraine prophylaxis: A randomized placebo-controlled study. Ann Indian Acad Neurol 2015; 18: 45-48.

11. Verma A, Srivastava D, Kumar A, Singh V. Levetiracetam in migraine prophylaxis: a randomized placebo-controlled study in a rural medical institute in northern India. Clin Neuropharmacol 2013; 36: 193-197.

12. Watkins AK, Gee ME, Brown JN. Efficacy and safety of levetiracetam for migraine prophylaxis: A systematic review. J Clin Pharm Ther 2018; 43: 467-475.

13. Headache Classification Committee of the International Headache Society (IHS) The International Classification of Headache Disorders, 3rd edition. Cephalalgia 2018; 38: 1-211.

14. Ashrafi MR, Salehi S, Malamiri RA, et al. Efficacy and safety of cinnarizine in the prophylaxis of migraine in children: a double-blind placebo-controlled randomized trial. Pediatr Neurol 2014; 51: 503-508.

15. Tonekaboni SH, Ghazavi A, Fayyazi A, et al. Prophylaxis of childhood migraine: topiramate versus propranolol. Iran J Child Neurol 2013; 7: 9-14

16. Miller GS. Efficacy and Safety of Levetiracetam in Pediatric Migraine. Headache 2004; 44: 238-243.

17. Cutrer FM, Limmroth V, Moskowitz MA. Possible mechanisms of valproate in migraine prophylaxis. Cephalalgia 1997; 17: 93-100.

18. Shahien R, Beiruti K. Preventive agents for migraine: focus on the antiepileptic drugs. J Cent Nerv Syst Dis 2012; 4: 37-49. 